\title{
Autophagy-related gene 7 is downstream of heat shock protein 27 in the regulation of eye morphology, polyglutamine toxicity, and lifespan in Drosophila
}

\author{
Shih-Fen Chen ${ }^{1 \dagger}$, Ming-Lun Kang ${ }^{1 \dagger}$, Yi-Chun Chen ${ }^{1+}$, Hong-Wen Tang ${ }^{3 \dagger}$, Cheng-Wen Huang ${ }^{1,4}$, Wan-Hua Li ${ }^{1}$, \\ Chun-Pu Lin ${ }^{2}$, Chao-Yung Wang ${ }^{5}$, Pei-Yu Wang ${ }^{4^{*}}$, Guang-Chao Chen ${ }^{3^{*}}$ and Horng-Dar Wang ${ }^{1,2^{*}}$
}

\begin{abstract}
Background: Autophagy and molecular chaperones both regulate protein homeostasis and maintain important physiological functions. Atg7 (autophagy-related gene 7) and Hsp27 (heat shock protein 27) are involved in the regulation of neurodegeneration and aging. However, the genetic connection between Atg7 and Hsp27 is not known.

Methods: The appearances of the fly eyes from the different genetic interactions with or without polyglutamine toxicity were examined by light microscopy and scanning electronic microscopy. Immunofluorescence was used to check the effect of Atg7 and Hsp27 knockdown on the formation of autophagosomes. The lifespan of altered expression of Hsp27 or Atg7 and that of the combination of the two different gene expression were measured.
\end{abstract}

Results: We used the Drosophila eye as a model system to examine the epistatic relationship between Hsp27 and Atg7. We found that both genes are involved in normal eye development, and that overexpression of Atg7 could eliminate the need for Hsp27 but Hsp27 could not rescue Atg7 deficient phenotypes. Using a polyglutamine toxicity assay (41Q) to model neurodegeneration, we showed that both Atg7 and Hsp27 can suppress weak, toxic effect by 41 Q, and that overexpression of Atg7 improves the worsened mosaic eyes by the knockdown of Hsp27 under 41Q. We also showed that overexpression of Atg7 extends lifespan and the knockdown of Atg7 or Hsp27 by RNAi reduces lifespan. RNAiknockdown of Atg7 expression can block the extended lifespan phenotype by Hsp27 overexpression, and overexpression of Atg7 can extend lifespan even under Hsp27 knockdown by RNAi.

Conclusions: We propose that Atg7 acts downstream of Hsp27 in the regulation of eye morphology, polyglutamine toxicity, and lifespan in Drosophila.

Keywords: Atg7, Hsp27, Neurodegeneration, Lifespan, Drosophila

\section{Background}

The aging process results from imbalanced homeostasis combined with accumulating macromolecular damage due to different intrinsic and environmental stresses [1-3]. Protein homeostasis is important in maintaining physiological function to protect against cellular degeneration

\footnotetext{
* Correspondence: pywang@nccu.edu.tw; gcchen@gate.sinica.edu.tw; hdwang@life.nthu.edu.tw

${ }^{\dagger}$ Equal contributors

${ }^{4}$ Institute of Neuroscience, National Chengchi University, 64, Section 2, ZhiNan Road, Taipei, 11605, Taiwan

Full list of author information is available at the end of the article
}

[4]. Autophagy and molecular chaperones are two defensive systems utilized to uphold cellular protein quality and homeostasis $[5,6]$.

Macroautophagy (herein called autophagy) is a cellular, catabolic process that breaks down and recycles macromolecules and organelles under starvation conditions. Autophagy function is executed by a series of autophagy related genes $(A t g)$ which are evolutionarily conserved from yeast to mammals [7]. Autophagy participates in many physiological functions including aging and neurodegeneration $[8,9]$, and mounting evidence demonstrates that autophagy participates in the regulation of

\section{Biomed Central}


lifespan in different species [10-12]. In C. elegans, lossof-function of bec-1/Atg6 or RNA interference-mediated depletion of Atg-7 or Atg-12 inhibits the extended lifespan in daf-2 mutants [13, 14], and the knockdown of bec-1 or Atg7 by RNAi abolishes dietary restrictionmediated longevity in eat-2 mutants [15]. In addition, mutations in Atg1, Atg7, Atg18, and bec-1 reduce lifespan in C. elegans [16]. In Drosophila, Atg7-null mutants are short-lived and hypersensitive to starvation and oxidative stress [17], and the neuronal overexpression of Atg8a regulates lifespan and tolerance to oxidative stress [18]. Atg7 is an E1-like enzyme and is important for the membrane elongation of the autophagosome [7]. Atg7 deficient mice exhibit polyubiquitinated protein accumulation and neurodegeneration [19] and higher levels of polyubiquitinated proteins have been detected in the aging $\operatorname{Atg} 7$ mutant fly head [17]. Autophagy also protects against neurodegeneration [20] and the induction of autophagy by the reduction of TOR (target of rapamycin) activity reduces polyglutamine toxicity in both fly and mouse [21]. Suppression of basal autophagy in the central nervous system causes neurodegeneration in Atg7 conditional knockout mice [19, 22].

Molecular chaperones modulate protein re-folding and facilitate the degradation of denatured proteins. Molecular chaperones are also implicated in several physiological functions: autophagy, neurodegeneration, stress tolerance, and aging [23-25]. Heat shock protein 27 (Hsp27) is a member of the ATP-independent, small heat shock protein family. Hsp27 null mutants exhibit decreased lifespan and reduced starvation tolerance [26], while the overexpression of $H s p 27$ increases lifespan and enhances stress resistance in Drosophila [27, 28]. Overexpression of $H s p 27$ prevents cellular polyglutamine toxicity and rescues the mosaic eyes induced by mild polyglutamine toxicity $[27,29]$.

Both $H s p 27$ and Atg7 are involved in maintaining protein quality and modulating lifespan and neurodegeneration. However, the interaction between Hsp27 and Atg7 is unknown. We report here that Atg7 is downstream of Hsp27 in the regulation of eye morphology, polyglutamine toxicity, and lifespan in Drosophila. The levels of Hsp27 and Atg7 both regulate eye morphology and the polyglutamine toxicity of $41 \mathrm{Q}$. The overexpression of Atg7 rescues both the rough eye phenotype resulting from knockdown of $\mathrm{Hsp} 27$ as well as the more severe mosaic eye phenotype induced by the knockdown of $H s p 27$ under 41Q toxicity. In addition, the expression of Atg7 regulates lifespan in Drosophila and the enhanced lifespan seen with the overexpression of $H s p 27$ requires the expression of Atg7. Together we provide several lines of genetic evidence linking Hsp27 to Atg7 in the modulation of eye morphology, polyglutamine toxicity, and lifespan regulation.

\section{Methods}

\section{Fly strains and maintenance}

The RNAi lines were obtained from Vienna Drosophila RNAi Center (VDRC), UAS-hsp27 $7^{R N A i}$ (\#40530), UAShsp22 $2^{R N A i}$ (\#43632), UAS-atg1 $1^{R N A i}$ (\#16133), UAS-atg4 ${ }^{R A i}$ (\#107317), UAS-atg5 $5^{R N A i} \quad$ (\#104461), UAS-atg7 $7^{R N A i}$ (\#45560), UAS-atg8a $a^{R N A i} \quad$ (\#43096), UAS-atg8a $a^{R N A i}$ (\#43097), UAS-atg9 $9^{R N A i} \quad(\# 10045), \quad$ UAS-atg12 $2^{R N A i}$ (\#102362), UAS-atg18 ${ }^{R N A i}$ (\#105366). GMR-Gal4; UAS$41 Q$ and GMR-Gal4/Cyo; UAS-63Q were provided by Dr. Parsa Kazemi-Esfarjani. To generate UAS-Atg7 transgenic flies, the EST clone RE27292 containing the fulllength Atg7 was used to amplify the coding sequence by the primers (forward: 5'-GTACTCGAGAAGCAA AACATGAGCACGG-3' and reverse: 5'-CATAGATCTATCCTCGTCGCT ATCGGA-3') and subcloned into the $X h o I$ and BglII sites of the transgenic vector, pINDY6 [28]. The resultant construct was verified by DNA sequencing to confirm that no mutations derived from PCR amplification were made, and injected into $w^{1118}$ eggs for the generation of UAS-Atg7 transgenic flies. All flies were maintained on standard fly food as described in Liu et al. [30] and incubated at $25^{\circ} \mathrm{C}, 65 \%$ humidity, in a $12 \mathrm{~h} / 12 \mathrm{~h}$ light/dark-cycle fly incubator.

\section{Fly eye image}

Two-day-old flies of the different types were anaesthetized by carbon dioxide on a porous platform and the eye images were taken by light microscopy (SMZ1500, Nikon). For the scanning electron micrograph, the fly was fixed on a copper stage and the fly eye image was acquired by scanning electron microscopy (TM-1000, Hitachi). For each fly line, a total of more than 86 eye images from at least three independent crosses were examined.

\section{RT-PCR and real-time PCR}

Total RNA was prepared from about 20 flies of each specific allele and homogenized in $1 \mathrm{ml}$ Trizol solution. Equal amounts $(1 \mu \mathrm{g})$ of each DNase I-treated RNA were reversetranscribed to cDNA with MMLV reverse transcriptase (Promega). The cDNAs were used as templates for RTPCR or real-time PCR as described in Liu et al. [30]. The information of the primers is available upon request.

\section{Lifespan and starvation assays}

For the lifespan assay, all the fly lines have been outcrossed with $w^{1118}$ as described previously [31]. The newly eclosed flies of each allele were collected by sex with 30 flies per vial, maintained at $25^{\circ} \mathrm{C}, 65 \%$ humidity in a $12 \mathrm{~h} / 12 \mathrm{~h}$ light/dark-cycle fly incubator and transferred to a new vial every 3 or 4 days until all were dead. The statistical significance was calculated by log rank test. At least three independent measurements were performed for each experiment. 
For the starvation assay, newly eclosed flies of each type were collected by sex with 20 flies per vial and recovered overnight. Next day the flies were transferred to the vials with $1 \%$ agar and transferred to new agar vials daily. The numbers of the dead flies were recorded every 4 hours until all were dead. The statistical significance was calculated by student's $t$ test.

\section{Immunofluorescence}

GFP-NLS-marked Atg7 or Hsp27 RNAi knockdown clones in the larval fat body were generated by heat shock-independent FLP/FRT induction as described previously [32, 33]. FLP/FRT method allows to examine the mitotic GFP-NLS-marked RNAi knockdown clones surrounded by the control cells that do not incorporate the RNAi knockdown in the same tissue under the same condition [33]. Fat bodies from early third instar larva cultured in standard fly food with yeast paste (fed condition) or in dishes containing $20 \%$ sucrose only (starvation condition) for $4 \mathrm{hr}$ were dissected and fixed with $4 \%$ paraformaldehyde and then examined by confocal laser scanning microscope (LSM510; Carl Zeiss Inc.) equipped with a 63x Plan-Apochromat (NA1.4) objective lens.

\section{Results}

Autophagy-related gene 7 is downstream of heat shock protein $\mathbf{2 7}$ in the regulation of Drosophila eye phenotype

Protein homeostasis plays an important role in lifespan and stress response $[1,2]$. Heat shock protein 27 (Hsp27) has been shown to regulate lifespan and response to different stresses [26-28]. Autophagy-related gene 7 (Atg7) is required for normal lifespan and tolerance to starvation and oxidation [17]. However, the genetic interaction between $H s p 27$ and Atg7 is unknown. We examined the effects of altering Hsp27 and Atg7 expression in the Drosophila eye using the GMR-Gal4 driver followed by the analyses of eye morphology utilizing scanning electron microscopy and light microscopy. Overexpression of Hsp27 or Atg7 results in a normal eye phenotype and regular ommatidia shape as seen in the GMR-Gal4 control flies (Figure 1, A-A", B-B", D-D"). Interestingly, knockdown expression of either $\mathrm{Hsp} 27$ or Atg7 by expression of interfering RNAs using GMR-Gal4 results in similar rough eye phenotypes with fused and enlarged ommatidia (Figure 1, C-C", E-E"). Overexpression of Atg7 in the Hsp27 knockdown background fully rescues the rough eye phenotype of the Hsp27 knockdown (Figure 1, F-F”). However, overexpression of $H s p 27$ in the Atg7 knockdown background fails to rescue the rough eye phenotype of the Atg7 knockdown (Figure 1, G-G"). These results suggest that Atg7 is located downstream of Hsp27 in the regulation of Drosophila eye morphology. To further confirm that Hsp27 and Atg7 function in the same pathway controlling eye phenotype, we examined whether there is any additive effect on fly eye morphology by either the cooverexpression or co-knockdown of Hsp27 and Atg7. The overexpression of both Hsp27 and Atg7 in combination produces a normal eye phenotype, similar to the overexpression of $\mathrm{Hsp} 27$ or Atg7 alone (Figure 1, B-B", DD", H-H"). The simultaneous knockdown of Hsp27 and Atg7 does not further deteriorate the rough eye phenotype when compared to the effects of either gene alone (Figure 1, C-C", E-E", I-I"), implying that Hsp27 and Atg7 operate in the same pathway. These data provide the first evidence that Atg7 is downstream of $H s p 27$ in the regulation of Drosophila eye morphology.

\section{Knockdown of other autophagy-related genes and heat shock protein 22 does not result in a rough eye phenotype in Drosophila}

To determine whether the rough eye phenotype is specific to Atg7, or whether it represents a general effect of altering autophagy, the effects of knockdown of additional autophagy-related genes were examined by using GMR-Gal4 and none of these displayed the rough eye phenotype (Figure 2, Figure 1, E-E”). Knockdown of Atg1 shows a normal eye phenotype (Figure 2, A-A", Figure 1, A-A"), while knockdown of other autophagyrelated genes: $\operatorname{Atg}$ 4, 5, 8, 9, 12, 18 displayed subtle eye color phenotypes but had no effect on the ommatidia structure (Figure 2, B-G, B'-G, B"-G”). These data suggests that the rough eye phenotype resulting from Atg7 knockdown is Atg7-specific and not involved in the alteration of other autophagy-related genes. Similarly, to examine whether the rough eye phenotype is specific to Hsp 27 knockdown, we tested the effects of knockdown of Hsp22, another known lifespan modulation gene [34], by GMR-Gal4 and did not observe any effects on the eye like that of Hsp27 knockdown (Figure 1, C-C"). Q-PCR analysis confirms that there is reduced expression of Atg and Hsp22 genes in the RNAi knockdown experiments (data not shown). Thus the rough eye phenotype is specific to the knockdown of either Atg7 or Hsp27.

\section{Knockdown of Atg7 but not Hsp27 blocks starvation- induced autophagosome formation}

To verify that the knockdown of $\operatorname{Atg} 7$ by $U A S-A \operatorname{tg} 7^{R N A i}$ from VDRC can affect starvation-induced autophagy, we generated $U A S-A \operatorname{tg} 7^{R N A i}$ clones in the fat-body by using the FLP/FRT method [32, 33] and examined the distribution of mcherry-Atg8a puncta. The distribution of mcherry-Atg8a is in a uniformly diffuse structure under optimal feeding conditions (Figure 3, B, J) and becomes localized to punctate structure under starvation conditions (Figure 3, F, N). Under starvation conditions, the GFP-NLS clones with the Atg7 knockdown, where the cells are circled by dotted line, display a reduced number of mcherry-Atg8a puncta than the surrounding control clones without Atg7 


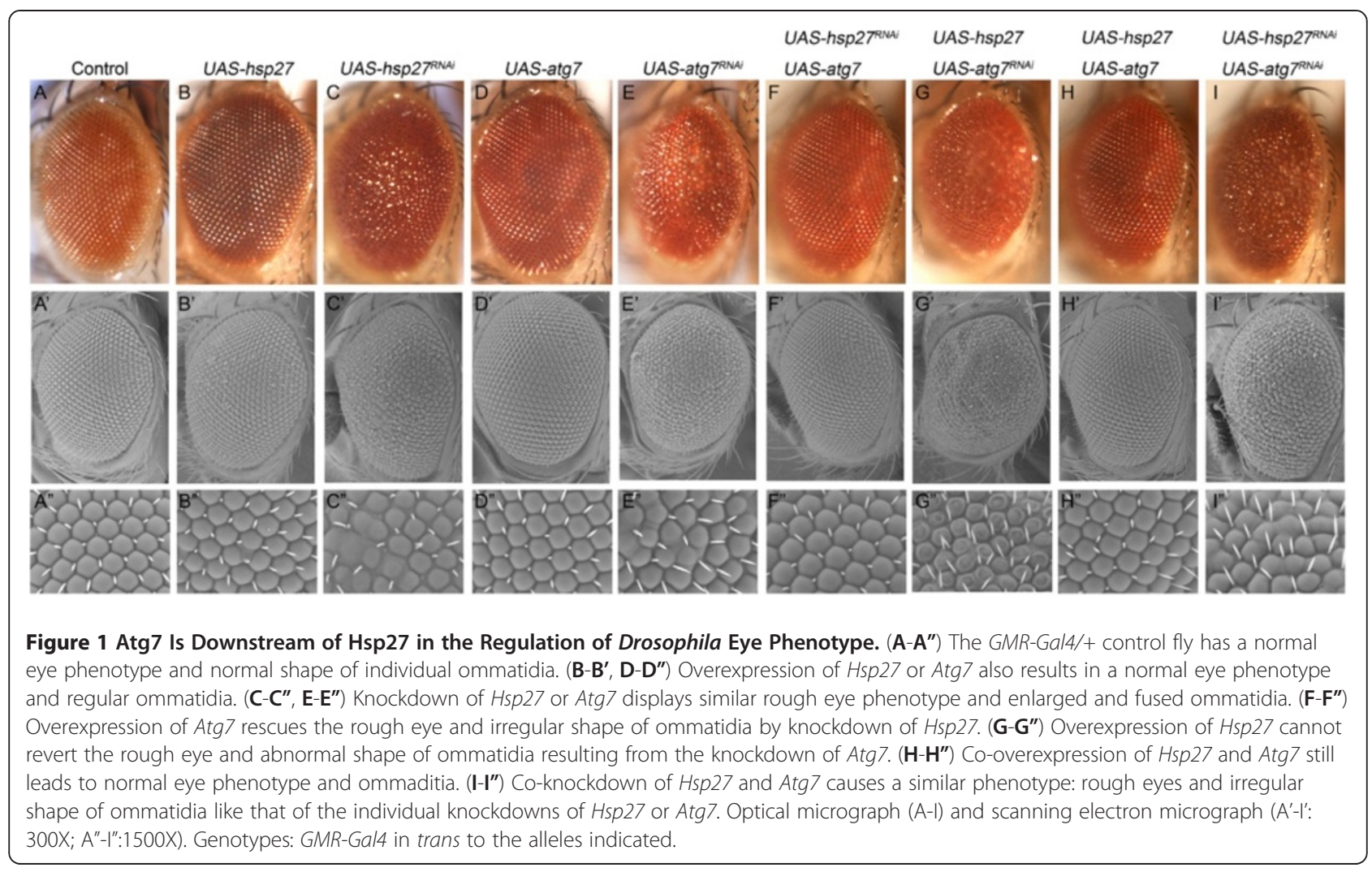

knockdown which have no GFP-NLS signal (Figure 3, E, F). These results demonstrate that knockdown of $\operatorname{Atg} 7$ by $U A S-\operatorname{Atg} 7^{R N A i}$ is able to block mcherry-Atg8a mediated autophagosome formation under starvation. To examine whether knockdown of $H s p 27$ can alter autophagosome formation, we also generated $U A S-H s p 27^{R N A i}$ clones in the fat body and inspected the distribution of mcherry-Atg8a puncta. Under starvation, the autophagosome formation

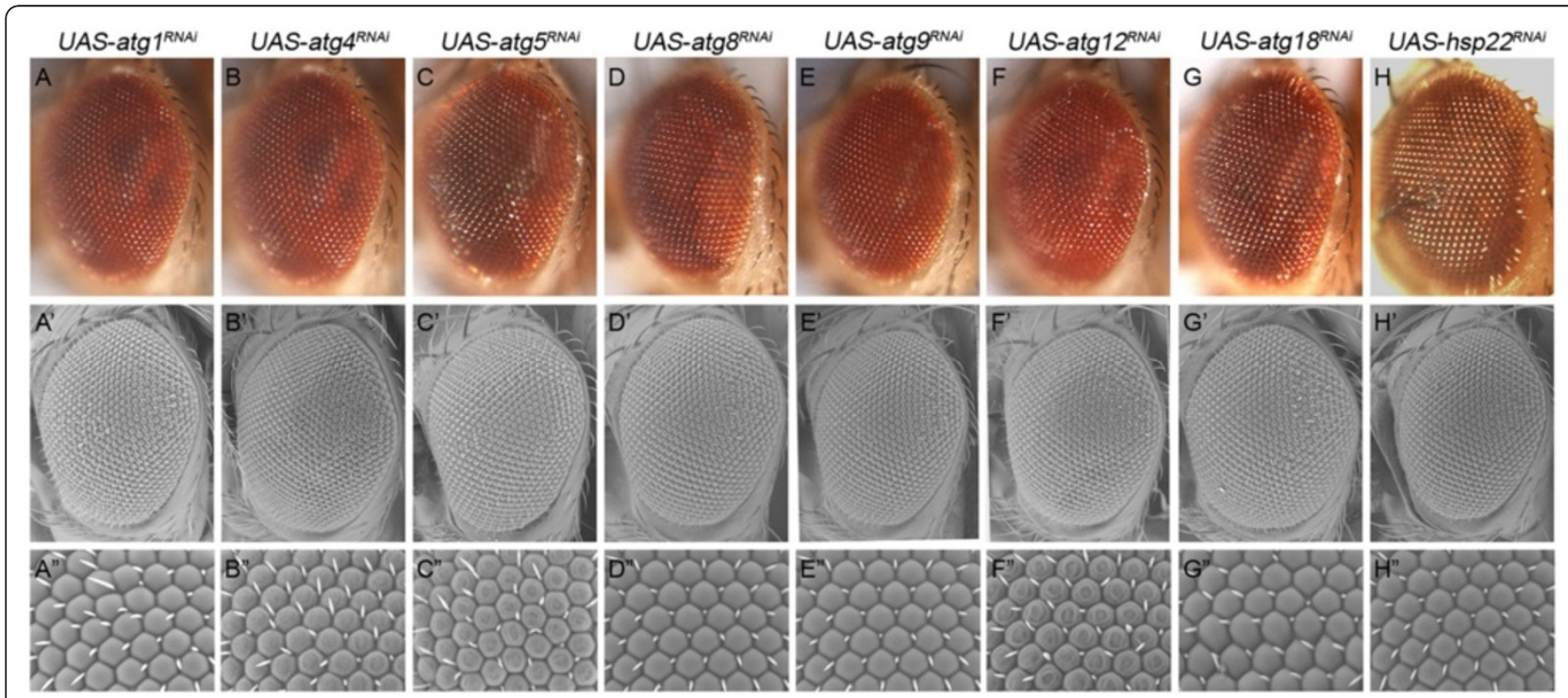

Figure 2 Knockdown of the Other Autophagy-related Genes and Heat Shock Protein 22 Do Not Result in Any Rough Eye Phenotype in Drosophila. RNAi knockdown of different autophagy-related genes by GMR-Gal4 show normal eye morphology and regular ommatidia (like the control in Figure 1, A-A". (A-A") Atg1, (B-B") Atg4, (C-C") Atg5, (D-D") Atg8a, (E-E") Atg9, (F-F") Atg12, (G-G") Atg18, and (H-H") Hsp22. Optical micrograph (A-H) and SEM (A'-H':300X; $\left.A^{\prime \prime}-H^{\prime \prime}: 1500 X\right)$. Genotypes: GMR-Gal4 in trans to the alleles indicated. 


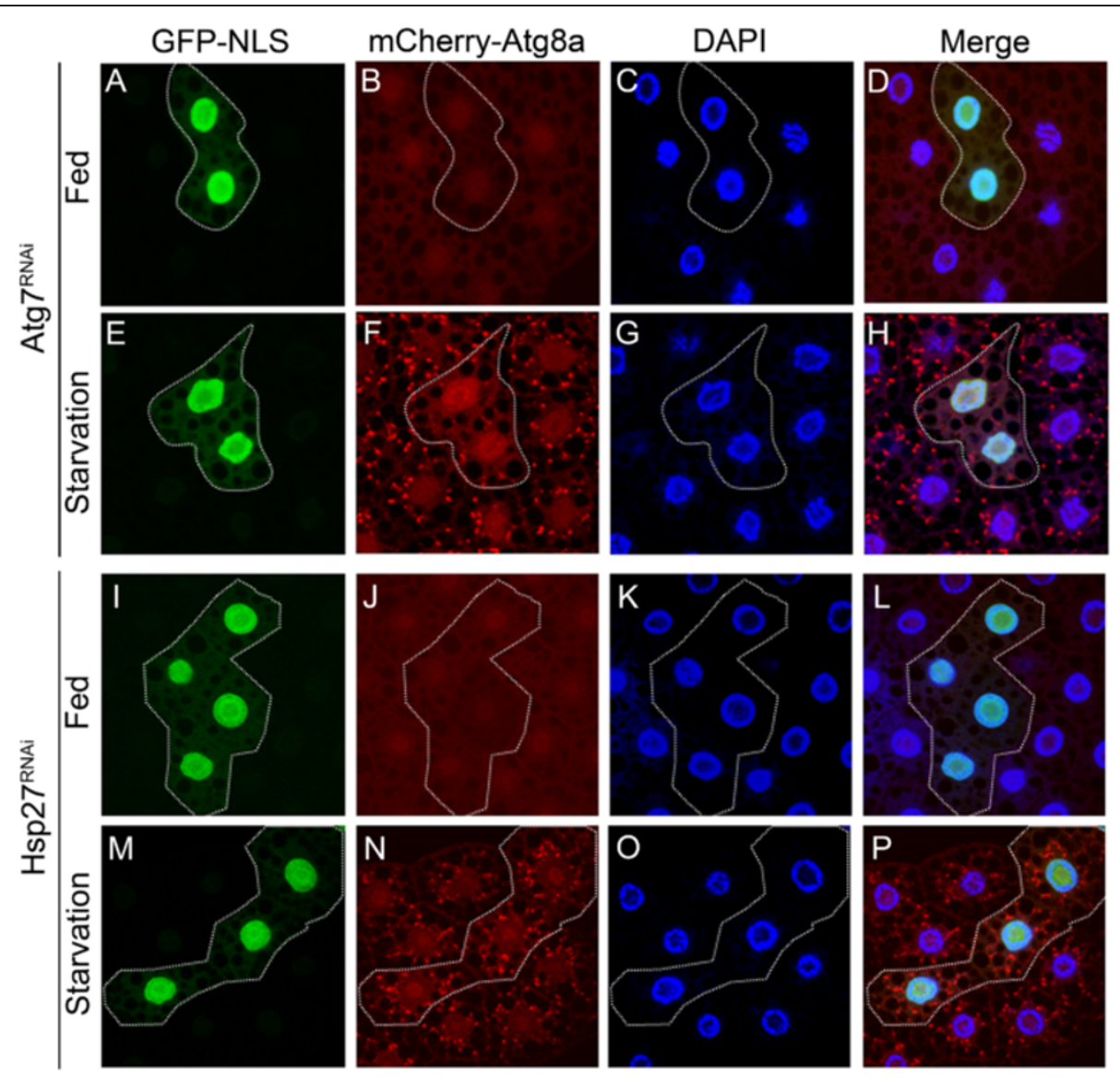

Figure 3 Starvation-induced Autophagosome Formation is Inhibited by RNAi-mediated Depletion of Atg7 but not Hsp27. (A, E, I, M) GFP-NLS labeled fat body cells circled by dotted line indicate the presence of UAS-Atg $7^{\text {RNA }}$ or UAS-Hsp2 $27^{\text {RNAi }}$ generated by the FLP/FRT method. The cells outside of the circled dotted line are used as the control cells without UAS-Atg $7^{\text {RNAi }}$ or UAS-Hsp27 $7^{R N A i}$. (B, F, J, N) The distribution patterns of mcherry-Atg8a are shown under either fully-fed or starvation conditions. (C, G, K, O) The fat body cells are stained with DAPI. (D, L) The picture $D$ is merged from panels $A, B, C$ and $L$ is merged from panels $I, J, K$ under nutrient-rich conditions. (H, P) Picture $H$ is merged from panels $E, F, G$ and $\mathrm{P}$ is merged from $\mathrm{M}, \mathrm{N}, \mathrm{O}$ under starvation conditions. The distribution of mcherry-Atg8a puncta is dramatically altered in starved fat body cells $(F, N)$ compared to those under nutrient-rich conditions (B, J). GFP-labeled cells expressing Atg7-RNAi markedly suppress mCherry-Atg8a puncta formation (F), but not in that of Hsp27 knockdown (N).

indicated by mcherry-Atg8a puncta is not altered by comparing the GFP-NLS marked Hsp27 RNAi knockdown clones, which are circled by dotted line, to the surrounding control clones without GFP-NLS signal and no Hsp27 RNAi knockdown (Figure 3, M, N). The data indicate that Hsp27 knockdown does not block the mcherry-Atg8a mediated autophagosome formation under starvation. The notion is consistent with the previous data since knockdown of $\operatorname{Atg} 8$ does not result in the rough eye as the knockdown of Hsp27, suggesting that Hsp27 and Atg8 do not function in the same genetic pathway.

Atg7 and $H s p 27$ attenuate the mild polyglutamine toxicity of $41 \mathrm{Q}$ but cannot rescue longer polyglutamine tract toxicity by $63 Q$

Overexpression of $H s p 27$ can rescue the mosaic eye phenotype resulting from mild polyglutamine (41Q)- induced toxicity but not the rough eye phenotype resulting from severe polyglutamine (127Q) toxicity [27]. Since Atg7 acts downstream of $H s p 27$ in the eye, we were interested in determining whether the overexpression of $A \operatorname{tg} 7$ would also only rescue mild polygutamine phenotypes. As with $H s p 27$, the overexpression of Atg7 rescues the mosaic eye phenotype caused by 41Q (Figure 4, A, B, D) but cannot rescue the more severe, rough eye phenotypes resulting from the longer polyglutamine tract of 63Q (Figure 4, G, $\mathrm{H}, \mathrm{J})$. The knockdown of either Hsp27 or Atg7 enhances the pigmentation phenotype observed in the eye of flies expressing 41Q. Interestingly only the knockdown of Atg7, but not that of Hsp27, enhances the eye morphology phenotype (rough eye) in combination with 41Q overexpression (Figure 4, C, E). The knockdown of Hsp27 or $\operatorname{Atg} 7$ does not exacerbate the rough eye phenotypes of 63Q (Figure 4, I). Interestingly, the overexpression of 
Atg7 partially rescues the more dramatic mosaic eye phenotype induced by Hsp27 knockdown in the $41 \mathrm{Q}$ background (Figure 4, C, F), supporting the idea that Atg7 is downstream of Hsp27 in the alleviation of $41 \mathrm{Q}$ toxicity. However, the combination of the overexpression of Atg7 and knockdown of Hsp27 do not change the rough eye phenotype of 63Q (Figure 4, L).

\section{Atg7 regulates lifespan and is required for Hsp27- mediated extended lifespan in Drosophila}

Hsp27 levels are likely to regulate Drosophila lifespan since Hsp27 overexpression extends Drosophila lifespan [27, 28] while the knockout Hsp27 mutant is short-lived [26]. The knockdown of Hsp27 by either $h s$-Gal4, or $d a-$ Gal4 exhibits reduced $H s p 27$ levels and displays a $20 \%$ $(P<0.001)$, and $27 \%(P<0.001)$ decrease in mean lifespan, respectively (Figure 5, A - D; Additional file 1: Table S1). Since Atg7 is downstream of Hsp27 in the regulation of eye morphology and mild polyglutamine toxicity, and Atg7 null mutants display shortened lifespan [17], we tested whether Hsp27-mediated enhanced lifespan requires Atg7. Atg7 overexpression by hs-Gal4 shows a robust increase in $A \operatorname{tg} 7$ transcripts relative to control flies and increases the mean lifespan by about $11 \%(P<0.01)$ relative to the control flies (Figure $5, \mathrm{E}$ and G; Additional file 2: Table S2). Conversely, knockdown of Atg7 by hs-Gal4 exhibits reduced levels of Atg7 transcripts and decreases mean lifespan by about $10 \%$ $(P<0.01)$ when compared to the control flies (Figures $5 \mathrm{~F}$ and $\mathrm{H}$; Additional file 2: Table S2). These results indicate that like Hsp27, Atg7 levels also regulate Drosophila lifespan.

It has been shown that neuronal overexpression of Atg8a by appl-Gal4 extends Drosophila lifespan and increases resistance to starvation [18]. To test whether neuronal overexpression of Atg7 enhances lifespan and starvation resistance, $A \operatorname{tg} 7$ was overexpressed in neurons using appl-Gal4, resulting in increases of $12 \%$ $(P<0.001)$ in mean lifespan and $18 \%(P<0.01)$ in starvation resistance (Figure 5, I; Additional file 2: Table S2 and Additional file 3:Table S4). In addition, the simultaneous overexpression of Atg7 and knockdown of $H s p 27$ results in flies that exhibit a $21 \%(P<0.001)$ extension in mean lifespan (Figure 5, J). Conversely, the flies possessing both knockdown of Atg7 and overexpressing $H s p 27$ display a reduction of $27 \%(P<0.001)$ in mean lifespan relative to the control flies (Figure 5, J; Additional file 4 : Table S3). To further demonstrate that Atg7 functions downstream of $H s p 27$, we carried out the locomotion assay to measure the climbing activity of the flies with the different combination of overexpression and knockdown of Atg7 and Hsp27 along with the control flies under paraquat-induced oxidative stress. Similar to the lifespan result, the flies with simultaneous overexpression of Atg7 and knockdown of Hsp27 displayed significantly better climbing activity $(42 \%, P \leq 0.001)$ than that of the control flies (22\%), and the flies with simultaneous knockdown of Atg7 and overexpression of Hsp27 exhibited a significantly lowered locomotion activity $(15 \%, P \leq 0.01)$ than that of the control flies 


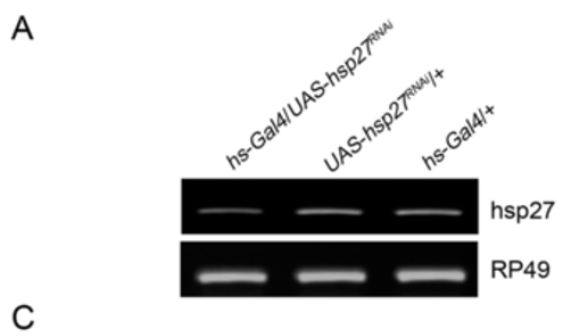

C

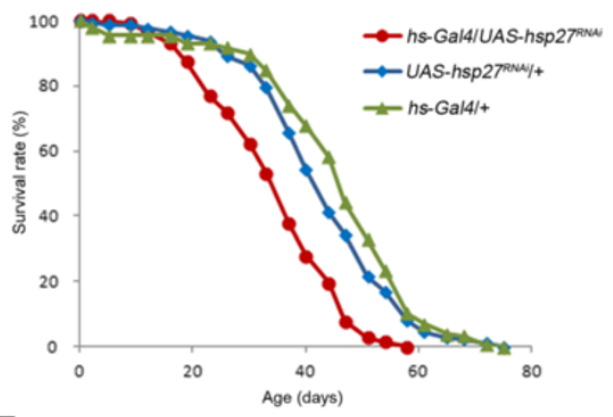

E

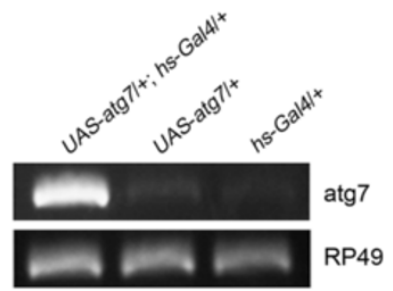

G

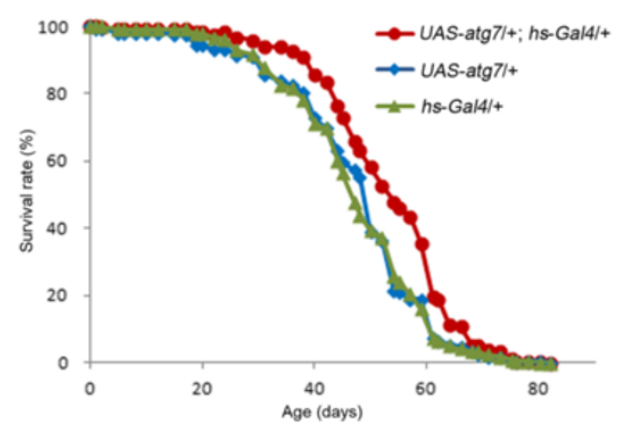

I

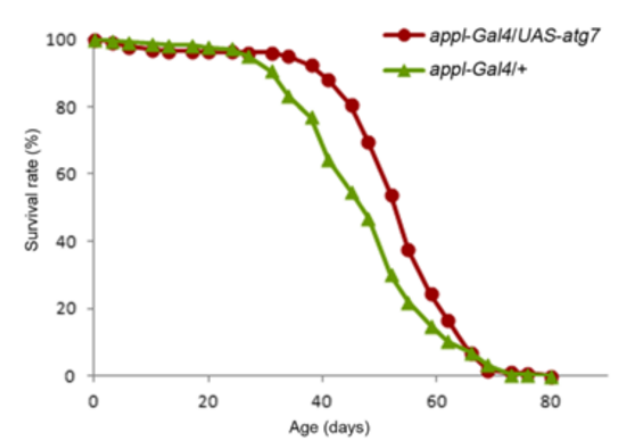

B

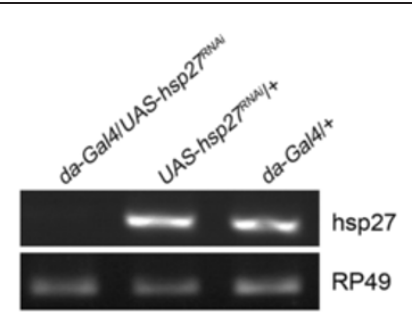

D

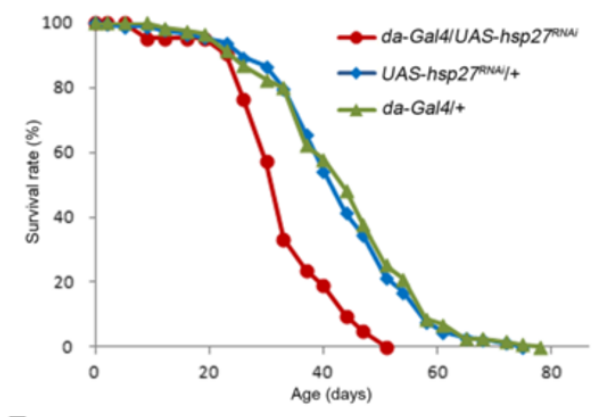

$\mathrm{F}$

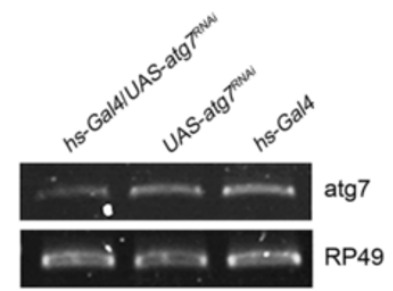

$\mathrm{H}$
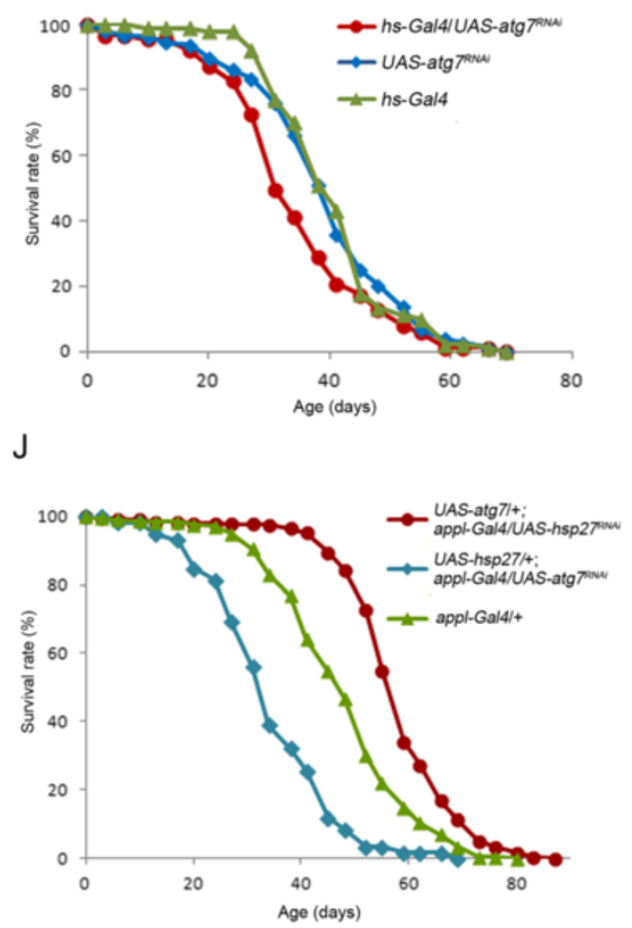

Figure 5 (See legend on next page.) 
(See figure on previous page.)

Figure 5 Atg7 Is Downstream of Hsp27 in the Regulation of Drosophila Lifespan. (A, B, E, F) RT-PCR verifies that the transcript levels of Hsp27 and Atg7 are altered upon Gal4 induction. (C, D) RNAi knockdown of Hsp27 by hs-Gal4 and da-Gal4 both reduce Drosophila lifespan. (G) Overexpression of Atg7 by hs-Gal4 increases Drosophila lifespan. (H) Knockdown of Atg7 by hs-Gal4 decreases Drosophila lifespan. (I) Neuronal overexpression of Atg7 by appl-Gal4 enhances Drosophila lifespan. (J) Overexpression of Atg7 along with knockdown of Hsp27 by appl-Gal4 displays extended lifespan. On the other hand, simultaneous knockdown of Atg7 and overexpression of Hsp27 exhibits reduced lifespan.

(Additional file 5: Figure S1). The climbing activity data in accordance with the lifespan data supports our hypothesis that Atg7 acts downstream of Hsp27. Taken together, these results indicate that as seen with Drosophila eye morphology and polyglutamine toxicity, Atg7 also acts downstream of $\mathrm{Hsp} 27$ in regulating lifespan.

\section{Discussion}

Hsp 27 and Atg7 are both implicated in the processes of aging and neurodegeneration. In this report, we provide several lines of evidence to show that $\operatorname{Atg} 7$ is downstream of $H s p 27$ in the regulation of eye morphology, polyglutamine toxicity, and lifespan. Autophagy-related genes are conserved among different species [7, 35]. Each of the identified Atgs has a role in autophagy, but their roles in other processes remains largely unclear.

In the examination of eye phenotype, we observed that the knockdown of either $H s p 27$ or Atg7 exhibited similar rough eye phenotypes. These effects appear to be specific to these particular molecules since the knockdown of other Atgs (Atg1, Atg4, Atg5, Atg8a, Atg9, Atg12, and Atg18) or $H s p 22$ does not produce a similar, rough eye phenotype. The ability of $\operatorname{Atg} 7$ to rescue the phenotype induced by $H s p 27$ knockdown also suggests that a unique interaction exists between Hsp27 and Atg7. A recent study indicates that knockdown of Atg7 by GMRGal4 on X chromosome causes retinal degeneration [36]. In addition, the rhabdomeres were shown degenerated in the aged $\operatorname{atg} 7^{d 77}$ mutant flies [37]. Both support our finding that RNAi knockdown of Atg7 results in rough eye in Drosophila.

Autophagy serves to protect against neurodegenerative diseases [20] and aberrations in autophagy have been implicated in neurodegeneration [38]. In both fly and mouse models, induction of autophagy by inhibiting mTOR ameliorates polyglutamine toxicity [21]. And in humans, a polymorphism study of more than 900 European Huntington's disease patients revealed that one variant of $\operatorname{Atg} 7\left(\operatorname{Atg} 7^{V 471 A}\right)$ is statistically correlated with early onset of Huntington's disease [39]. These findings suggest that a specific function of Atg7 is to attenuate polyglutamine toxicity and support our findings that Atg7 rescues polyglutamine toxicity by $41 \mathrm{Q}$ in Drosophila. Hsp27 has also been shown to reduce cellular polyglutamine toxicity [29] and the overexpression of Hsp27 in Drosophila rescues the pigmentation defects induced by 41Q [27]. Several lines of evidence suggest that heat shock proteins may rely upon autophagy to reduce polyglutamine toxicity. For example, the anti-polyglutamineaggregation activity of $\mathrm{HspB} 7$, one of the human small heat shock proteins, was substantially diminished in Atg5-deficient cells [40]. In addition, it is possible that the small heat shock protein HspB8-Bag3 complex enhance Htt43Q degradation via autophagy since the treatment of the Htt43Q transfected HEK-293T and COS1 cells with an autophagy inhibitor significantly reduced HspB8-Bag3-mediated Htt43Q degradation [41]. Furthermore, it was recently suggested that the small heat shock protein HspB7 assists in the loading of misfolded proteins or aggregates in autophagosomes [42]. Together, these findings indicate that autophagy is downstream of small heat shock proteins and support our results that $A \operatorname{tg} 7$ is downstream of $H s p 27$.

The inhibition of autophagy results in decreased lifespan. Atg7 activity is essential for the longevity resulting from either reduced insulin signaling or caloric restriction in which depletion of Atg7 was found to block the longevity phenotypes of both daf-2 and eat-2 mutants $[13,15]$. Our data showed that RNAi knockdown of Atg7 by hs-Gal4, starting from embryonic to adulthood stage, results in a shortened lifespan similar to that of the Drosophila Atg7 null mutant [17]. Loss-of-function mutations in Atg7 as well as Atg1, Atg18, and Beclin-1 shorten lifespan in C. elegans [16]. Several autophagy mutants including $\operatorname{Atg} 7$ were identified chronologically short-lived in a yeast genetic screen [43]. However, it should be noted that not all autophagy genes are linked to aging and Atg7 is one of the conserved Atg genes that is involved in the regulation of aging in most species [9]. Conversely, the induction of autophagy increases lifespan. The induction of autophagy by caloric restriction or reducing target of rapamycin (TOR) activity enhances lifespan [9] and the neuronal overexpression of Atg8a increases Drosophila lifespan [18]. We have found that the overexpression of Atg7 extends lifespan in Drosophila and that the neuronal overexpression of $\operatorname{Atg} 7$ is sufficient to reverse Hsp27-knockdown-mediated, shortened lifespan. Knockdown of Atg7 blocks Hsp27-mediated extended lifespan, again supporting the model that Atg7 acts downstream of Hsp27 in the regulation of lifespan. It has been reported that in adult flies, RNAi knockdown of Atg7 by Geneswitch-Actin-Gal4 did not show reduced lifespan [44]. This discrepancy may be due to the different Gal4 drivers used and that the knockdown of $\operatorname{Atg} 7$ 
occurring only during adulthood is insufficient to cause shortened lifespan since autophagy activity is known to be tightly regulated during development.

Yet we cannot exclude that chaperone-mediated autophagy (CMA) is involved in the connection between Hsp27 and Atg7. CMA is a specific cargo delivery process to the lumen of the lysosome, mediated by Hsc70, Hsp90, and the lysosome-associated membrane protein type 2A (LAMP-2A) $[45,46]$. However, a recent study in Drosophila shows that the co-chaperone Starvin assists in the coordination of Hsc70 and HspB8 through chaperone-assisted selective autophagy, which is distinct from CMA, to depose damaged filamin for muscle maintenance [47]. It is possible that Hsp27 may function through chaperone-assisted selective autophagy linking to Atg7.

\section{Conclusion}

In summary, our finding sheds new insight in the linkage of Hsp27 to Atg7 in the regulation of eye morphology, polyglutamine toxicity, and lifespan. The information provides a new aspect in the understanding how Hsp27 may connect to Atg7 to modulate certain physiological functions.

\section{Additional files}

Additional file 1: Table S1. A summary of lifespan by the knockdown of Hsp27 in Drosophila.

Additional file 2: Table S2. A summary of lifespan by the overexpression or knockdown of Atg7 in Drosophila.

Additional file 3: Table S4. A summary of starvation stress response by overexpression of Atg7 in Drosophila.

Additional file 4: Table S3. A summary of lifespan resulting from simultaneous overexpression and knockdown of different combinations of Atg7 and Hsp27 in Drosophila.

Additional file 5: Figure S1. The flies with simultaneous overexpression of Atg7 and knockdown of Hsp27 display better climbing activity than those with overexpression of Hsp27 and knockdown of Atg7 under paraquat-induced oxidative stress. The climbing index for each strain: appl-Gal4/+(the control fly): $21.8 \pm 0.02 \%(n=195) ;$ UAS-hsp27/+; applGal4/UAS-atg $7^{\text {RNAi: }}: 14.7 \pm 0.02 \%(n=123)$; UAS-atg7/4; appl-Gal4/UAShsp2 $27^{\text {RAA }}: 42.4 \pm 0.01 \%(n=175)$. ( $n$ is the total fly number from the four independent assays.). ( ${ }^{* *} p<0.01,{ }^{* * *} p<0.001$ ).

\section{Abbreviations}

Atg: autophagy-related gene; Hsp: heat shock protein.

\section{Competing Interests}

The authors declare that they have no competing interests.

\section{Acknowledgements}

We thank Drs. Theodore Brummel, Micheline Laurent, William Ja, Pankaj Kapahi, and Chiou-Hwa Yuh for the critical reading and suggestions for the manuscript. We thank the fly lines from VDRC and Bloomington stock center, GMR-Gal4; UAS-41Q and GMR-Gal4/Cyo; UAS-63Q from Dr. Parsa KazemiEsfarjani. We thank the funding support from National Tsing Hua University/ Chang Gung Memorial Hospital collaboration grant (101N2754E1), NTHU/ McKay Hospital collaboration grant (99N2903E1), and National Science Council (100-2311-B-007-006-) to Dr. Horng-Dar Wang, National Science
Council grant (100-2311-B-004-001-MY3) to Dr. Pei-Yu Wang, and Academia Sinica (AS-99-TP-B09) to Dr. Guang-Chao Chen. We are indebted for the funding support from Brain Research Center (101N2060E1) by Dr. Ann-Shyn Chiang at NTHU, the fly import assistance from Fly Core Taiwan by Dr. Chau-Ti Ting, and the transgenic fly support by Dr. Y. Henry Sun at Academia Sinica, Taipei, Taiwan.

\section{Author details}

${ }^{1}$ Institute of Biotechnology, National Tsing Hua University, 101, Section 2, Kuang-Fu Road, HsinChu, 30013, Taiwan. ²Department of Life Science, National Tsing Hua University, 101, Section 2, Kuang-Fu Road, HsinChu, 30013, Taiwan. ${ }^{3}$ Institute of Biological Chemistry, 128, Section 2, Academia Road, Nankang, Taipei, 115, Taiwan. ${ }^{4}$ Institute of Neuroscience, National Chengchi University, 64, Section 2, Zhi-Nan Road, Taipei, 11605, Taiwan. ${ }^{5}$ Second Section of Cardiology, Department of Internal Medicine, Chang Gung Memorial Hospital at Linkou, Chang Gung University College of Medicine, Taoyuan, Taiwan.

\section{Authors' contributions}

S-F Chen, M-L Kang, Y-C Chen, H-W Tang, C-W Huang, W-H Li, and C-P Lin carried out the experiments and analyzed the data; P-Y Wang, G-C Chen and $\mathrm{H}-\mathrm{D}$ Wang designed the experiments, analyzed the data, and together with C-Y Wang discussed the data and wrote the manuscript. All authors read and approved the final manuscript.

Received: 2 February 2012 Accepted: 23 May 2012

Published: 23 May 2012

\section{References}

1. Haigis MC, Yankner BA: The aging stress response. Mol Cell 2010, 40(2):333-344

2. Koga H, Kaushik S, Cuervo AM: Protein homeostasis and aging: The importance of exquisite quality control. Ageing Res Rev 2011, 10(2):205-215.

3. Taylor RC, Dillin A: Aging as an event of proteostasis collapse. Cold Spring Harb Perspect Biol 2011, 3(5):a004440.

4. Douglas PM, Dillin A: Protein homeostasis and aging in neurodegeneration. J Cell Biol 2010, 190(5):719-729. 2010/09/08 ed.

5. Tyedmers J, Mogk A, Bukau B: Cellular strategies for controlling protein aggregation. Nat Rev Mol Cell Biol 2010, 11(11):777-788.

6. Wong E, Cuervo AM: Integration of clearance mechanisms: the proteasome and autophagy. Cold Spring Harb Perspect Biol 2011, 2(12): a006734.

7. He C, Klionsky DJ: Regulation mechanisms and signaling pathways of autophagy. Annu Rev Genet 2009, 43:67-93.

8. Kroemer G, Marino G, Levine B: Autophagy and the integrated stress response. Mol Cell 2010, 40(2):280-293.

9. Rubinsztein DC, Marino G, Kroemer G: Autophagy and aging. Cell 2011 146(5):682-695.

10. Chang YY, Neufeld TP: Autophagy takes flight in Drosophila. FEBS Lett 2010, 584(7):1342-1349.

11. Cuervo AM, Bergamini E, Brunk UT, Droge W, Ffrench M, Terman A: Autophagy and aging: the importance of maintaining "clean" cells. Autophagy 2005, 1(3):131-140.

12. Madeo F, Tavernarakis N, Kroemer G: Can autophagy promote longevity? Nat Cell Biol 2010, 12(9):842-846.

13. Hars ES, Qi H, Ryazanov AG, Jin S, Cai L, Hu C, Liux LF: Autophagy regulates ageing in C. elegans. Autophagy 2007, 3(2):93-95.

14. Melendez A, Talloczy Z, Seaman M, Eskelinen EL, Hall DH, Levine B: Autophagy genes are essential for dauer development and life-span extension in C. elegans. Science 2003, 301(5638):1387-1391.

15. Jia K, Levine B: Autophagy is required for dietary restriction-mediated life span extension in C. elegans. Autophagy 2007, 3(6):597-599.

16. Toth ML, Sigmond T, Borsos E, Barna J, Erdelyi P, Takacs-Vellai K, Orosz L, Kovacs AL, Csikos G, Sass M, Vellai T: Longevity pathways converge on autophagy genes to regulate life span in Caenorhabditis elegans. Autophagy 2008, 4(3):330-338.

17. Juhasz G, Erdi B, Sass M, Neufeld TP: Atg7-dependent autophagy promotes neuronal health, stress tolerance, and longevity but is dispensable for metamorphosis in Drosophila. Genes Dev 2007, 21(23):3061-3066. 
18. Simonsen A, Cumming RC, Brech A, Isakson P, Schubert DR, Finley KD: Promoting basal levels of autophagy in the nervous system enhances longevity and oxidant resistance in adult Drosophila. Autophagy 2008, 4(2):176-184.

19. Komatsu M, Waguri S, Chiba T, Murata S, Iwata J, Tanida I, Ueno T, Koike M, Uchiyama Y, Kominami E, Tanaka K: Loss of autophagy in the central nervous system causes neurodegeneration in mice. Nature 2006, 441(7095):880-884

20. Garcia-Arencibia M, Hochfeld WE, Toh PP, Rubinsztein DC: Autophagy, a guardian against neurodegeneration. Semin Cell Dev Biol 2010, 21(7):691-698

21. Ravikumar B, Vacher C, Berger Z, Davies JE, Luo S, Oroz LG, Scaravilli F, Easton DF, Duden R, O'Kane CJ, Rubinsztein DC: Inhibition of mTOR induces autophagy and reduces toxicity of polyglutamine expansions in fly and mouse models of Huntington disease. Nat Genet 2004, 36(6):585-595.

22. Hara T, Nakamura K, Matsui M, Yamamoto A, Nakahara Y, Suzuki-Migishima R, Yokoyama M, Mishima K, Saito I, Okano H, Mizushima N: Suppression of basal autophagy in neural cells causes neurodegenerative disease in mice. Nature 2006, 441(7095):885-889.

23. Lanneau D, Brunet M, Frisan E, Solary E, Fontenay M, Garrido C: Heat shock proteins: essential proteins for apoptosis regulation. J Cell Mol Med 2008, 12(3):743-761

24. Tower J: Heat shock proteins and Drosophila aging. Exp Geronto/ 2011, 46(5):355-362.

25. Voisine C, Pedersen JS, Morimoto Rl: Chaperone networks: tipping the balance in protein folding diseases. Neurobiol Dis 2010, 40(1):12-20.

26. Hao X, Zhang S, Timakov B, Zhang P: The Hsp27 gene is not required for Drosophila development but its activity is associated with starvation resistance. Cell Stress Chaperones 2007, 12(4):364-372

27. Liao PC, Lin HY, Yuh CH, Yu LK, Wang HD: The effect of neuronal expression of heat shock proteins 26 and 27 on lifespan, neurodegeneration, and apoptosis in Drosophila. Biochem Biophys Res Commun 2008, 376(4):637-641.

28. Wang HD, Kazemi-Esfarjani P, Benzer S: Multiple-stress analysis for isolation of Drosophila longevity genes. Proc Natl Acad Sci U S A 2004, 101(34):12610-12615.

29. Wyttenbach A, Sauvageot O, Carmichael J, Diaz-Latoud C, Arrigo AP, Rubinsztein DC: Heat shock protein 27 prevents cellular polyglutamine toxicity and suppresses the increase of reactive oxygen species caused by huntingtin. Hum Mol Genet 2002, 11(9):1137-1151.

30. Liu YL, Lu WC, Brummel TJ, Yuh CH, Lin PT, Kao TY, Li FY, Liao PC, Benzer S, Wang HD: Reduced expression of alpha-1,2-mannosidase I extends lifespan in Drosophila melanogaster and Caenorhabditis elegans. Aging Cell 2009, 8(4):370-379

31. Wang CT, Chen YC, Wang YY, Huang MH, Yen TL, Li H, Liang CJ, Sang TK, Ciou SC, Yuh CH, Wang CY, Brummel TJ, Wang HD: Reduced neuronal expression of ribose-5-phosphate isomerase enhances tolerance to oxidative stress, extends lifespan, and attenuates polyglutamine toxicity in Drosophila. Aging Cell 2012, 11(1):93-103.

32. Tang HW, Wang YB, Wang SL, Wu MH, Lin SY, Chen GC: Atg1-mediated myosin II activation regulates autophagosome formation during starvation-induced autophagy. EMBO J 2011, 30(4):636-651.

33. Theodosiou NA, Xu T: Use of FLP/FRT system to study Drosophila development. Methods 1998, 14(4):355-365.

34. Morrow G, Samson M, Michaud S, Tanguay RM: Overexpression of the small mitochondrial Hsp22 extends Drosophila life span and increases resistance to oxidative stress. FASEB J 2004, 18(3):598-599.

35. Kourtis N, Tavernarakis N: Autophagy and cell death in model organisms. Cell Death Differ 2009, 16(1):21-30.

36. Midorikawa R, Yamamoto-Hino M, Awano W, Hinohara Y, Suzuki E, Ueda R, Goto S: Autophagy-dependent rhodopsin degradation prevents retinal degeneration in Drosophila. J Neurosci 2010, 30(32):10703-10719.

37. Wang T, Lao U, Edgar BA: TOR-mediated autophagy regulates cell death in Drosophila neurodegenerative disease. J Cell Biol 2009, 186(5):703-711.

38. Marino G, Madeo F, Kroemer G: Autophagy for tissue homeostasis and neuroprotection. Curr Opin Cell Biol 2011, 23(2):198-206.

39. Metzger S, Saukko M, Van Che H, Tong L, Puder Y, Riess O, Nguyen HP: Age at onset in Huntington's disease is modified by the autophagy pathway: implication of the V471A polymorphism in Atg7. Hum Genet 2010, 128(4):453-459.
40. Vos MJ, Zijlstra MP, Kanon B, van Waarde-Verhagen MA, Brunt ER, Oosterveld-Hut HM, Carra S, Sibon OC, Kampinga HH: HSPB7 is the most potent polyQ aggregation suppressor within the HSPB family of molecular chaperones. Hum Mol Genet 2010, 19(23):4677-4693.

41. Carra S, Seguin SJ, Lambert H, Landry J: HspB8 chaperone activity toward poly(Q)-containing proteins depends on its association with Bag3, a stimulator of macroautophagy. J Biol Chem 2008, 283(3):1437-1444.

42. Vos MJ, Zijlstra MP, Carra S, Sibon OC, Kampinga HH: Small heat shock proteins, protein degradation and protein aggregation diseases. Autophagy 2011, 7(1):101-103.

43. Matecic M, Smith DL, Pan X, Maqani N, Bekiranov S, Boeke JD, Smith JS: A microarray-based genetic screen for yeast chronological aging factors. PLoS Genet 2010, 6(4):e1000921.

44. Ren C, Finkel SE, Tower J: Conditional inhibition of autophagy genes in adult Drosophila impairs immunity without compromising longevity. Exp Gerontol 2009, 44(3):228-235.

45. Arias E, Cuervo AM: Chaperone-mediated autophagy in protein quality control. Curr Opin Cell Biol 2011, 23(2):184-189.

46. Dice JF: Chaperone-mediated autophagy. Autophagy 2007, 3(4):295-299.

47. Arndt V, Dick N, Tawo R, Dreiseidler M, Wenzel D, Hesse M, Furst DO, Saftig P, Saint R, Fleischmann BK, Hoch M, Hohfeld J: Chaperone-assisted selective autophagy is essential for muscle maintenance. Curr Biol 2010, 20(2):143-148.

\section{doi:10.1186/1423-0127-19-52}

Cite this article as: Chen et al:: Autophagy-related gene 7 is downstream of heat shock protein 27 in the regulation of eye morphology, polyglutamine toxicity, and lifespan in Drosophila. Journal of Biomedical Science 2012 19:52.

\section{Submit your next manuscript to BioMed Central and take full advantage of:}

- Convenient online submission

- Thorough peer review

- No space constraints or color figure charges

- Immediate publication on acceptance

- Inclusion in PubMed, CAS, Scopus and Google Scholar

- Research which is freely available for redistribution 